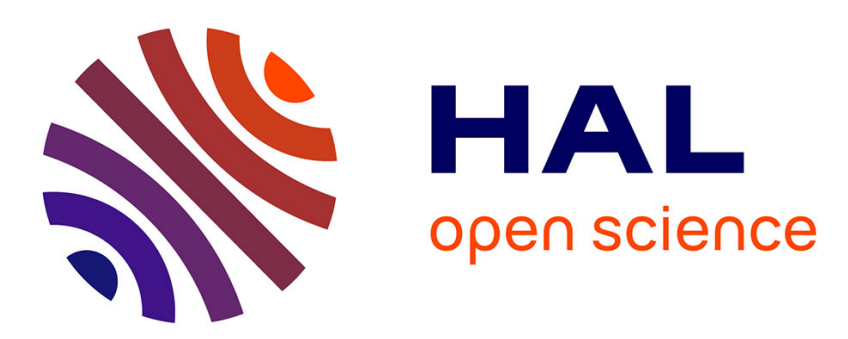

\title{
Full order unknown inputs observers design for delay systems
}

Mohamed Darouach

\section{To cite this version:}

Mohamed Darouach. Full order unknown inputs observers design for delay systems. Jun 2006, pp.6. hal-00120903

\section{HAL Id: hal-00120903 https://hal.science/hal-00120903}

Submitted on 18 Dec 2006

HAL is a multi-disciplinary open access archive for the deposit and dissemination of scientific research documents, whether they are published or not. The documents may come from teaching and research institutions in France or abroad, or from public or private research centers.
L'archive ouverte pluridisciplinaire HAL, est destinée au dépôt et à la diffusion de documents scientifiques de niveau recherche, publiés ou non, émanant des établissements d'enseignement et de recherche français ou étrangers, des laboratoires publics ou privés. 


\title{
Full Order Unknown Inputs Observers Design for Delay Systems
}

\author{
M.Darouach \\ CRAN-CNRS (UMR 7039), UHP-NancyI \\ IUT de Longwy, 186, Rue de Lorraine \\ 54400 COSNES et ROMAIN \\ FRANCE \\ e-mail darouach@iut-longwy.uhp-nancy.fr
}

\begin{abstract}
This paper presents the full order observers design for linear time delay systems with unknown inputs. A new method generalizing those existing in the literature is developed, the unknown inputs are present in both the state and the measurement equations. Conditions for the existence of these observers are given and sufficient conditions for the stability independent of delays are derived using linear matrix-inequality (LMI) formulation. The independent of internal delay and independent of delay cases are also presented. A numerical example is presented to illustrate our approach.
\end{abstract}

Keywords. Full order, Delay systems, Unknown inputs, LMI, Existence conditions, Stability, Independent of delay.

\section{INTRODUCTION}

Time delay systems are frequently encountered in industrial applications, such as chemical processes, thermal and hydraulic systems [1], [2], [3]. Recently , a great interest has been devoted to the stability, the stabilization, the control and the observers design for delay systems. The control is often realized with the assumption that the entire state vector is available trough output measurement. Since this is not generally true in the practice, it is necessary to design observers which produce an estimate of this state vector.

Observers for delay systems with known inputs problem has been investigated over the years and several design methods have been proposed ( see [4]-[7] and references therein ). The observers for systems with unknown inputs are of great interest in the failure detection and the control of systems in presence of disturbances [8]. The problem of unknown inputs observers for linear systems with delay has been recently considered [9]-[11], [13]. In [9] the method of unknown input observer for free delay systems has been extended to linear time delay systems by using a polynomial approach. The dynamic gain Luenberger-type observer was proposed in [10]. In [13] a new method for the observers design for linear time delay systems with unknown inputs has been proposed, however the proposed observer is dependent of derivative of the output. In all these works, the unknown inputs are only present in the state equation.

In this paper, a new method for the full orders observers design for time-delay systems with unknown inputs is presented. The unknown inputs are present in both the state and the measurement equations. Conditions for the existence of these observers are derived. Their stability is studied and a linear matrix-inequality (LMI) formulation is presented. The particular cases where the observer is independent of internal delay and of delay are also considered.

\section{Problem formulation}

Consider the following linear differential-delay system with unknown inputs

$$
\begin{aligned}
\dot{x}(t) & =A x(t)+A_{d} x(t-\tau)+F d(t)+B u(t) \\
y(t) & =C x(t)+G d(t)
\end{aligned}
$$

with the initial state $x(\theta)=\phi(\theta), \forall \theta \in[-\tau, 0]$, and where $x(t) \in \mathbb{R}^{n}$ is the state vector, $u(t) \in \mathbb{R}^{m}$ is the known input vector, $d(t) \in \mathbb{R}^{q}$ is the unknown input vector, and $y(t) \in \mathbb{R}^{p}$ is the measurement vector output, $\tau$ is the time-delay of the system satisfying $0 \leq \tau(t) \leq \tau_{0}$ and $0 \leq \dot{\tau}(t) \leq \tau_{1}<1$. Matrices $A, A_{d}, B, C, F$, and $G$ are real and of appropriate dimensions.

Consider the following full order observer for system (1)

$$
\begin{aligned}
& \dot{\zeta}(t)=N \zeta(t)+N_{d} \zeta(t-\tau)+D y(t)+D_{d} y(t-\tau)+H u(t) \\
& \widehat{x}(t)=\zeta(t)+E y(t)
\end{aligned}
$$

where the initial condition $\zeta(\theta)=\rho(\theta), \forall \theta \in[-\tau, 0], \zeta(t) \in \mathbb{R}^{n}$ is the state vector and $\widehat{x}(t) \in \mathbb{R}^{n}$ is the estimate of $x(t)$. $N, N_{d}, D, D_{d}, H$, and $E$ are unknown matrices of appropriate dimensions, which must be determined such that $\widehat{x}(t)$ asymptotically converges to $x(t)$.

Now define $e(t)$ as the error between $x(t)$ and its estimate $\widehat{x}(t)$ as

$$
e(t)=x(t)-\widehat{x}(t)=x(t)-\widehat{z}(t)=\Phi x(t)-\zeta(t)-E G d(t)
$$


where $\Phi=L-E C$. The dynamic of this error is given by:

$$
\begin{aligned}
\dot{e}(t)=N e(t)+N_{d} e(t-\tau)+(\Phi A- & N \Phi-D C) x(t)+\left(\Phi A_{d}-N_{d} \Phi-D_{d} C\right) x(t-\tau)+ \\
& (N E G-D G+\Phi F) d(t)+\left(N_{d} E G-D_{d} G\right) d(t-\tau)+(\Phi B-H) u(t)-E G \dot{d}(t)
\end{aligned}
$$

Then it is easy to prove the following theorem.

Theorem 1. Observer(2) will estimate (asymptotically) $x(t)$ if the following conditions hold:

i) $\dot{e}(t)=N e(t)+N_{d} e(t-\tau)$ is asymptotically stable

ii) $\Phi A-N \Phi-D C=0$

iii) $\Phi A_{d}-N_{d} \Phi-D_{d} C=0$

iv) $N E G-D G+\Phi F=0$

v) $N_{d} E G-D_{d} G=0$

vi) $E G=0$

vii) $H=\Phi B$

\section{MAIN RESUlts}

\section{A. General case}

In this section a new method is presented to design observer (2) for system (1) and its existence and stability conditions are given.

From theorem 1, the design of the observer(2) is reduced to find the matrices $N, N_{d}, D, D_{d}, E$, and $H$ so that conditions $i$ ) - vii) are satisfied.

Now using the definition of $\Phi$, equations $i i)$-vi) can be written as

$$
\begin{gathered}
N=A-\left[\begin{array}{lll}
K_{1} & K_{2} & E
\end{array}\right]\left[\begin{array}{c}
C \\
0 \\
C A
\end{array}\right] \\
N_{d}=A_{d}-\left[\begin{array}{lll}
K_{1} & K_{2} & E
\end{array}\right]\left[\begin{array}{c}
0 \\
C \\
C A_{d}
\end{array}\right]
\end{gathered}
$$

under the constraint

$$
\left[\begin{array}{lll}
K_{1} & K_{2} & E
\end{array}\right] \mathcal{M}_{1}=\mathcal{M}_{2}
$$

where $\mathcal{M}_{1}=\left[\begin{array}{ccc}G & 0 & 0 \\ 0 & G & 0 \\ C F & 0 & G\end{array}\right]$ and $\mathcal{M}_{2}=\left[\begin{array}{llll}F & 0 & 0 & 0\end{array}\right]$. The necessary and sufficient condition for the existence of the solution of (6) can then be given by the following lemma.

Lemma 1. The necessary and sufficient condition for the existence of the solution to (6) is

$$
\operatorname{rank}\left[\begin{array}{cc}
C F & 0 \\
G & 0
\end{array}\right]=\operatorname{rank}\left[\begin{array}{c}
G \\
F
\end{array}\right]+\operatorname{rank}(G)
$$

Proof. From the general solution of linear matrix equations [12], there exists a solution to (6) if and only if:

$$
\mathcal{M}_{2} \mathcal{M}_{1}^{+} \mathcal{M}_{1}=\mathcal{M}_{2}
$$

where $\mathcal{M}_{1}^{+}$is the generalized inverse matrix of $\mathcal{M}_{1}$.

Equation (8) is also equivalent to

$$
\operatorname{rank}\left[\begin{array}{c}
\mathcal{M}_{1} \\
\mathcal{M}_{2}
\end{array}\right]=\operatorname{rank}\left[\mathcal{M}_{1}\right]
$$

which leads to the condition (7)

Under (7), the solution of (6) is given by

$$
\left[\begin{array}{lll}
K_{1} & K_{2} & E
\end{array}\right]=\mathcal{M}_{2} \mathcal{M}_{1}^{+}+Z\left(I-\mathcal{M}_{1} \mathcal{M}_{1}^{+}\right)
$$

where $Z$ is an arbitrary matrix, which can be determined such that the observer is asymptotically stable. Now from the above results, matrices $N$ and $N_{d}$ are given by

$$
N=\mathcal{A}_{1}-Z \mathcal{B}_{1}
$$

and

$$
N_{d}=\mathcal{A}_{2}-Z \mathcal{B}_{2}
$$


where $\mathcal{A}_{1}=A-\mathcal{M}_{2} \mathcal{M}_{1}^{+}\left[\begin{array}{c}C \\ 0 \\ C A\end{array}\right], \mathcal{A}_{2}=A_{d}-\mathcal{M}_{2} \mathcal{M}_{1}^{+}\left[\begin{array}{c}0 \\ C \\ C A_{d}\end{array}\right], \mathcal{B}_{1}=\left(I-\mathcal{M}_{1} \mathcal{M}_{1}^{+}\right)\left[\begin{array}{c}C \\ 0 \\ C A\end{array}\right]$, and $\mathcal{B}_{2}=\left(I-\mathcal{M}_{1} \mathcal{M}_{1}^{+}\right)\left[\begin{array}{c}0 \\ C \\ C A_{d}\end{array}\right]$.

Under condition (7) and from the above results, the observer error dynamic can be written as

$$
\dot{e}(t)=N e(t)+N_{d} e(t-\tau)=\left(\mathcal{A}_{1}-Z \mathcal{B}_{1}\right) e(t)+\left(\mathcal{A}_{2}-Z \mathcal{B}_{2}\right) e(t-\tau)
$$

Now the problem of observer (2) design is reduced to the determination of the matrix parameter $Z$ such that condition i) of theorem 1 is satisfied.

Before giving the stability conditions of the obtained observer we can give the following lemma.

Lemma 2. Under condition (7) there exists a matrix parameter $Z$ such that $N$ is Hurwitz if and only if

$$
\operatorname{rank}\left[\begin{array}{cc}
\lambda I-A & -F \\
C & G
\end{array}\right]=n+\operatorname{rank}\left[\begin{array}{l}
G \\
F
\end{array}\right], \forall \lambda \in \mathbb{C}, \operatorname{Re}(\lambda) \geq 0
$$

Proof. From the expression of $N$, we have $N$ is Hurwitz if and only if the pair $\left(\mathcal{B}_{1}, \mathcal{A}_{1}\right)$ is detectable or equivalently

$$
\operatorname{rank}\left[\begin{array}{c}
\lambda I-\mathcal{A}_{1} \\
\mathcal{B}_{1}
\end{array}\right]=r, \forall \lambda \in \mathbb{C}, \operatorname{Re}(\lambda) \geq 0
$$

Now it is easy to see that (10) is equivalent to

$$
\operatorname{rank}\left[\begin{array}{cccc}
\lambda I-A & -F & 0 & 0 \\
C & G & 0 & 0 \\
0 & 0 & G & 0 \\
C A & C F & 0 & G
\end{array}\right]=\operatorname{rank} \mathcal{M}_{1}, \forall \lambda \in \mathbb{C}, \operatorname{Re}(\lambda) \geq 0
$$

Now, we have

$$
\begin{aligned}
& \operatorname{rank}\left[\begin{array}{cccc}
\lambda I-A & -F & 0 & 0 \\
C & G & 0 & 0 \\
0 & 0 & G & 0 \\
C A & C F & 0 & G
\end{array}\right]=\operatorname{rank}\left[\begin{array}{cc}
\lambda I-A & -\mathcal{M}_{2} \\
{\left[\begin{array}{c}
C \\
0 \\
C A
\end{array}\right]} & \mathcal{M}_{1}
\end{array}\right]=\operatorname{rank}\left[\begin{array}{cc}
I & \mathcal{M}_{2} \mathcal{M}_{1}^{+} \\
0 & I-\mathcal{M}_{1} \mathcal{M}_{1}^{+} \\
0 & \mathcal{M}_{1} \mathcal{M}_{1}^{+}
\end{array}\right]\left[\begin{array}{cc}
\lambda I-A & -\mathcal{M}_{2} \\
{\left[\begin{array}{cc}
C \\
0 \\
C A
\end{array}\right]} & \mathcal{M}_{1}
\end{array}\right] \\
& =\operatorname{rank}\left[\begin{array}{cc}
\lambda I-\mathcal{A}_{1} & 0 \\
\mathcal{B}_{1} & 0 \\
\mathcal{M}_{1} \mathcal{M}_{1}^{+}\left[\begin{array}{c}
C \\
0 \\
C A
\end{array}\right] & M_{1}
\end{array}\right]=\operatorname{rank}\left[\begin{array}{c}
\lambda I-\mathcal{A}_{1} \\
\mathcal{B}_{1}
\end{array}\right]+\operatorname{rank}\left(\mathcal{M}_{1}\right)
\end{aligned}
$$

where we have used the fact that $\mathcal{M}_{2}=\mathcal{M}_{2} \mathcal{M}_{1} \mathcal{M}_{1}^{+}$.

Which proves the lemma.

Now we can give the independent of delay conditions for the stability of the observer.

Theorem 2. Suppose that conditions (7) and(10) are satisfied. Then there exists an asymptotically stable unknown input observer of the form (2), if there exist matrices $0<P=P^{T}, 0<Q=Q^{T}$, and $Y$ satisfying the following LMI:

$$
\left[\begin{array}{cc}
P \mathcal{A}_{1}+\mathcal{A}_{1}^{T} P+Q-Y \mathcal{B}_{1}-\mathcal{B}_{1}^{T} Y^{T} & P \mathcal{A}_{2}-Y \mathcal{B}_{2} \\
\mathcal{A}_{2}^{T} P-\mathcal{B}_{2}^{T} Y^{T} & -Q_{\tau}
\end{array}\right]<0
$$

with $Q_{\tau}=\left(1-\tau_{1}\right) Q$, and the parameter matrix $Z$ is given by $Z=P^{-1} Y$.

Proof. Easy to prove from the use of the following Lyapunov-Krasovskii functional [6]:

$$
V(e, t)=e^{T}(t) P e(t)+\int_{t-\tau}^{t} e^{T}(\theta) Q e(\theta) d \theta
$$

where $P=P^{T}>0$ and $Q=Q^{T}>0$. By differentiating $V(e, t)$ along the solution of equation $\left.i\right)$ of theorem 1 we obtain

$$
\dot{V}(e, t)=e^{T}(t)\left[P N+N^{T} P+Q\right] e(t)+e^{T}(t) P N_{d} e(t-\tau)+e^{T}(t-\tau) N_{d}^{T} P e(t)-(1-\dot{\tau}) e^{T}(t-\tau) Q e(t-\tau)
$$

using the fact that $\dot{\tau} \leq \tau_{1}$ we obtain

$$
\dot{V}(e, t) \leq\left[\begin{array}{c}
e(t) \\
e(t-\tau)
\end{array}\right]^{T}\left[\begin{array}{cc}
P N+N^{T} P+Q & P N_{d} \\
N_{d}^{T} P & -Q_{\tau}
\end{array}\right]\left[\begin{array}{c}
e(t) \\
e(t-\tau)
\end{array}\right]
$$


If $\dot{V}(e, t)<0$, when $\left[\begin{array}{c}e(t) \\ e(t-\tau)\end{array}\right] \neq 0$ then $e(t) \longrightarrow 0$ as $t \longrightarrow \infty$ and $\left.i\right)$ of theorem 1 is satisfied. From $(12), \dot{V}(e, t)<0$

$$
\left[\begin{array}{cc}
P N+N^{T} P+Q & P N_{d} \\
N_{d}^{T} P & -Q_{\tau}
\end{array}\right]<0
$$

substituting the values of $N$ and $N_{d}$ in this LMI we obtain the result of the theorem.

If the delay $\tau$ is constant we obtain the following corollary.

corollary 1. Suppose that conditions (7) and (10) are satisfied. Then there exists an asymptotically stable unknown input observer of the form (2), if there exist matrices $0<P=P^{T}, 0<Q=Q^{T}$, and $Y$ satisfying the following LMI:

$$
\left[\begin{array}{cc}
P \mathcal{A}_{1}+\mathcal{A}_{1}^{T} P+Q-Y \mathcal{B}_{1}-\mathcal{B}_{1}^{T} Y^{T} & P \mathcal{A}_{2}-Y \mathcal{B}_{2} \\
\mathcal{A}_{2}^{T} P-\mathcal{B}_{2}^{T} Y^{T} & -Q
\end{array}\right]<0
$$

Then, the parameter matrix $Z=P^{-1} Y$.

\section{B. Independent of internal delay observers}

In this section we present the application of the above results to the particular case where the observer (2) is independent of internal delay (case where $N_{d}=0$ ). If $N_{d}=0$, the observer (2) becomes an independent of internal delay functional observer:

$$
\begin{aligned}
& \dot{\zeta}(t)=N \zeta(t)+D y(t)+D_{d} y(t-\tau)+H u(t) \\
& \widehat{x}(t)=\zeta(t)+E y(t)
\end{aligned}
$$

Define the following matrices:

$$
\mathcal{N}=\left[\begin{array}{llll}
A_{d} & F & 0 & 0
\end{array}\right]
$$

and

$$
\mathcal{M}=\left[\begin{array}{cccc}
0 & G & 0 & 0 \\
C & 0 & G & 0 \\
C A_{d} & C F & 0 & G
\end{array}\right]
$$

In this case conditions of lemma 1 and lemma 2 become

$$
\operatorname{rank}\left[\begin{array}{c}
\mathcal{M} \\
\mathcal{N}
\end{array}\right]=\operatorname{rank} \mathcal{M}
$$

and

$$
\operatorname{rank}\left[\begin{array}{ccccc}
\lambda I-A & 0 & -F & 0 & 0 \\
C & 0 & G & 0 & 0 \\
0 & C & 0 & G & 0 \\
C A & C A_{d} & C F & 0 & G
\end{array}\right]=\operatorname{rank} \mathcal{M}, \forall \lambda \in \mathbb{C}, \operatorname{Re}(\lambda) \geq 0
$$

Then we obtain

$$
\left[\begin{array}{lll}
K_{1} & K_{2} & E
\end{array}\right]=\mathcal{N M}^{+}+Z_{2}\left(I-\mathcal{M} \mathcal{M}^{+}\right)
$$

and

$$
N=A-\mathcal{N M}^{+}\left[\begin{array}{c}
C \\
0 \\
C A
\end{array}\right]-Z_{2}\left(I-\mathcal{M M}^{+}\right)\left[\begin{array}{c}
C \\
0 \\
C A
\end{array}\right]
$$

The matrix parameter $Z_{2}$ can be obtained from any pole placement method.

\section{Independent of delay observers}

In this section we present the application of the above results to the particular case where the observer (2) is independent of delay ( case where $N_{d}=0$ and $D_{d}=0$ ). This case corresponds to treat the delayed part of the system as a disturbance, and can be solved from the standard problem. In fact, in this case, observer (2) becomes

$$
\begin{aligned}
& \dot{\zeta}(t)=N \zeta(t)+D y(t)+H u(t) \\
& \widehat{x}(t)=\zeta(t)+E y(t)
\end{aligned}
$$

Then equation (6) can be written as 


$$
\left[\begin{array}{lll}
N & K_{1} & E
\end{array}\right] \Sigma_{1}=\Theta_{1}
$$

where $\Sigma_{1}=\left[\begin{array}{ccc}0 & G & 0 \\ C A_{d} & C F & G\end{array}\right]$ and $\Theta_{1}=\left[\begin{array}{ccc}A_{d} & F & 0\end{array}\right]$.

The conditions of lemma 1 and lemma 2 become

$$
\operatorname{rank}\left[\begin{array}{ccc}
0 & G & 0 \\
C A_{d} & C F & G \\
A_{d} & F & 0
\end{array}\right]=\operatorname{rank}\left[\begin{array}{ccc}
0 & G & 0 \\
C A_{d} & C F & G
\end{array}\right]
$$

and

$$
\operatorname{rank}\left[\begin{array}{cccc}
\lambda I-A & -A_{d} & -F & 0 \\
C & 0 & G & 0 \\
C A & C A_{d} & C F & G
\end{array}\right]=\operatorname{rank}\left[\begin{array}{cccc}
L & 0 & 0 & 0 \\
C & 0 & G & 0 \\
C A & C A_{d} & C F & G
\end{array}\right], \forall \lambda \in \mathbb{C}, \operatorname{Re}(\lambda) \geq 0
$$

These conditions are necessary and sufficient for the existence and stability of the independent of delay observers.

\section{NumericAl EXAMPLE}

To illustate the above results, consider the system of [13] with $A=\left[\begin{array}{cc}-1 & 0 \\ 0 & -2\end{array}\right], A_{d}=\left[\begin{array}{ll}1 & 0 \\ 0 & 1\end{array}\right], B=\left[\begin{array}{l}1 \\ 0\end{array}\right], F=\left[\begin{array}{l}1 \\ 2\end{array}\right]$, $G=0, C=\left[\begin{array}{ll}1 & 0\end{array}\right]$, and $\tau(t)$ satisfies $0 \leqslant \dot{\tau}(t) \leqslant \tau_{1}=0.2$. From the above results we obtain $N=\left[\begin{array}{cc}0 & 0 \\ 2 & -2\end{array}\right]-Z_{1}\left[\begin{array}{cc}1 & 0 \\ 0 & 0\end{array}\right]$ and $N_{d}=\left[\begin{array}{cc}0 & 0 \\ -2 & 1\end{array}\right]-Z_{1}\left[\begin{array}{ll}0 & 0 \\ 1 & 0\end{array}\right]$, where $Z_{1}=\left[\begin{array}{ll}K_{1} & K_{2}\end{array}\right]$. The determination of the parameter matrix $Z_{1}$ can be obtained by solving the LMI (11) of theorem 2, which gives $P=\left[\begin{array}{cc}54.1741 & 0 \\ 0 & 26.0086\end{array}\right], Q=\left[\begin{array}{cc}59.4594 & 0 \\ 0 & 55.8234\end{array}\right], Y=$ $\left[\begin{array}{cc}56.8167 & 0 \\ 52.0173 & -52.0173\end{array}\right]$ and the parameter matrix $Z_{1}=\left[\begin{array}{ll}K_{1} & K_{2}\end{array}\right]=P^{-1} Y=\left[\begin{array}{cc}1.0488 & 0 \\ 2 & -2\end{array}\right]$. Then we obtain $N=$ $\left[\begin{array}{cc}-1.0488 & 0 \\ 0 & -2\end{array}\right], N_{d}=\left[\begin{array}{ll}0 & 0 \\ 0 & 1\end{array}\right], E=F=\left[\begin{array}{l}1 \\ 2\end{array}\right], D=N E+K_{1}=\left[\begin{array}{c}0 \\ -2\end{array}\right], D_{d}=N_{d} E+K_{2}=0$, and $H=I-E C=\left[\begin{array}{c}0 \\ -2\end{array}\right]$.

\section{Conclusion}

In this paper a new method for the full order observers design for linear time-delay systems with unknown inputs has been developed. The obtained results generalize the existing results. Conditions for the existence of these observers are given. The sufficient conditions for the stability are derived using an LMI formulation, and necessary and sufficient conditions for the independent of internal delay and independent of delay observers are presented.

\section{REFERENCES}

1] S.I.Niculescu., Delay effects on stability, A robust control approach. Springer Verlag, 2001.

[2] M.Malek-Zavarei and M.Jamshidi., Time delay systems: analysis, optimization and applications. North-Holland, 1987.

[3] J.K.Hale and S.M.V.Lunel., Introduction to functional differential equations.New York: Springer-Verlag, 1993.

[4] A.E.Pearson and Y.A.Fiagbedzi, "An observer for time lag systems "IEEE Trans.Autom.Contr, vol.Ac-13, pp775-777, 1989.

[5] J.Leyva-Ramos and A.E.Pearson, "An asymptotic modal observer for linear autonomous time lag systems " IEEE Trans.Autom.Contr , vol.Ac-40, pp1291-1294, 1995.

[6] M.Darouach, "Linear functional observers for systems with delays in state variables "IEEE.Trans.Automat.Contr. , vol 46, pp491-496, 2001.

[7] M.Darouach, P.Pierrot and E.Richard, "Design of reduced-order observers without internal delays" IEEE.Trans.Automat.Contr. , vol 44, pp1711-1714, 1999.

[8] M.Darouach, M.Zasadzinski, and S.J.Xu, " Full-order observer for linear systems with unknown inputs " IEEE.Trans.Automat.Contr., vol 39, pp606-609, 1994.

[9] A.Fattouh, O.Sename, and J.M.Dion, "An unknown input observer design for linear time-delay systems" Proc. 38th IEEE Confer. on Decision and Control, pp4222-4227, Phoenix, Arizona, USA, 1999.

[10] O.Sename, "Unknown input robust observers for time-delay systems" Proc. 36th IEEE Confer. on Decision and Control, pp1629-1630, SanDiego, California, USA, 1997.

[11] O.Sename, A.Fattouh, and J.M.Dion, " Further results on unknown input observers design time-delay systems" Proc. 40th IEEE Confer. on Decision and Control, pp4635-4636, Orlando, Florida, USA, 2001.

[12] C.R.Rao and S.K.Mitra, Generalized inverse of matrices and its applications. Wiley, 1971.

[13] Y.M.Fu, G.R Duan, and S.M.Song, " Design of unknown input observer for linear time-delay systems " International journal of control, automation, and syst., vol.2, no 4, pp 530-535, 2004. 